\title{
Review of: "Asymmetric Arp2/3-mediated actin assembly facilitates clathrin-mediated endocytosis at stalled sites in genome-edited human stem cells"
}

Henri-François Renard

Potential competing interests: The author(s) declared that no potential competing interests exist.

This interesting study of Jin et al sheds a new light on how actin cytoskeleton contributes to clathrinmediated endocytosis (CME). Although the role of actin in CME has been studied for years (especially in yeast), it is not totally clear yet how the forces generated by actin cytoskeleton contribute to the formation and scission of endocytic pits, and how this depends on conditions (more or less membrane tension or turgor pressure) in mammalian cells. The central finding of this study highlights the fact that actin and actin nucleators (N-WASP, ARPC3) are recruited asymmetrically to CME sites, leading the authors to suggest a model where actin forces act as a "bottle opener" to facilitate vesicle scission in mammals. Undoubtedly, the strength of this study resides in the "high-throughput/systematic" way they study CME events, which offers a refined and statistical view of the situation. The authors combined genome-edition, advanced microscopy approaches (3-colours live-cell TIRF, 2-colours 3D-STORM) and cutting-edge computational tools based on machine-learning for image analysis.

Although one may consider this study as somewhat limited in terms of variety of approaches, these are very elegantly implemented, experiments are well conducted and the data really brings a new light. However, while the authors mention/hypothesize the role of membrane tension and forces generated by actin throughout their manuscript, the study lacks data including those parameters. Some additional and most likely challenging experiments might thus complete this already very nice study.

\section{Major comments:}

$\left.1^{\circ}\right)$ From the text and the models presented in Figure $5 e$, the authors suggest that the asymmetric actin assemblies at CME sites produce forces that push away laterally the endocytic pits, which assists the fission process. However, the amplitude of these forces is not explored in the current study. Maybe a first way to have access to this information would be to check if a displacement of the vesicular core of the CME pit relatively to its neck (marked with dynamin or BAR domain proteins such as SNX9 or amphiphysin, maybe?) is observed upon asymmetric actin assembly. Maybe other biophysical and computational approaches I am not a specialist of could also help? This would strengthen the manuscript.

$2^{\circ}$ ) The authors mention that actin assembly is necessary only in case of high local membrane tension, where it helps to complete scission. However, they did not provide experimental data connecting their observations to membrane tension. As they provide a statistical study on thousands of CME sites, it would 
have been very interesting to verify if changing membrane tension (by osmotic shock or stretching cells, or by changing substrate stiffness, for instance) modifies the proportion of CME sites with asymmetric actin assemblies, and if their dynamics is changed. As a corollary, it would be interesting to check if, in unperturbed conditions, the appearance of asymmetric actin assemblies on CME sites correlates with local membrane tension. I guess this is very challenging technically (maybe a nice opportunity to use probes such as Flipper-TR?).

\section{Minor comments:}

$\left.1^{\circ}\right)$ It is not clear why the authors use iPSCs.

$2^{\circ}$ ) Page 3, $\S 2$, line 3: wouldn't it be more appropriate to use mu2 rather than mu1? It is generally said that AP2M1 gene codes for mu2-adaptin. The use of mu1 may introduce confusion with mu1-adaptin of AP1 complex, encoded by AP1M1.

$3^{\circ}$ ) Page 7 , end of $\S 1$ : when discussing data presented in Figure 3 , the authors conclude that the observation of ARPC3 at early stages of CME corresponds most likely to unrelated actin structures present in the surroundings of CME sites. It is not clear if, in the subsequent analyses (Figure 4, Extended Data Figure 5), those CME events are taken into account as ARPC3-negative or N-WASP-negative CME sites or if they are just discarded from the analysis. Unless it is mentioned somewhere in the manuscript, it could be interesting that the authors clarify this point.

$\left.4^{\circ}\right)$ Page 10, §1: the authors propose a very interesting hypothesis according to which asymmetrical forces may twist the clathrin pit to favor scission. In a very recent NCB paper, Cheng et al observed a dynamindependent twist of the vesicle during the final stage of CME, that they call "super twist" (DOI: $10.1038 / \mathrm{s} 41556-021-00713-\mathrm{x}$ ). Is there any connection that could be established with this study? Could it be that the twist is allowed by the concerted actions of dynamin and asymmetric actin assemblies? Comments from authors on that point would be very interesting. In a future study, it would be nice to verify if the extent of the twist is different when actin is present or not (synergy between dynamin and actin?). 